\title{
Uterine Adenosarcoma with Low Grade Overgrowth and Smooth Muscle Differentiation Mimicking Leiomyosarcoma
}

\author{
Amal B ${ }^{1 *}$, karich ${ }^{1}$, Saadi $\mathrm{H}^{2}$, Kamaoui ${ }^{3}$ and Mimouni $\mathrm{A}^{2}$ \\ ${ }^{1}$ Department of pathology, Mohamed I University, Oujda, Morocco \\ 2Department of the gynecology, Mohamed I University, Oujda, Morocco \\ ${ }^{3}$ Department of radiology, Mohamed I University, Oujda, Morocco
}

Case Report

Volume 3 Issue 1

Received Date: April 27, 2018

Published Date: May 05, 2018

*Corresponding author: Amal Bennani, Department of pathology, Mohamed I University, Bp 6210. Assaada 60020 Oujda, Morocco, Tel: +212662781319; Email: bennaniamal@gmail.com

\section{Abstract}

Background: Adenosarcoma is a rare mixed mesodermal tumor of the uterus which is composed of benign glandular epithelium and malignant mesenchymal stroma.

Case Presentation: 59-year-old postmenopausal women, presented with vaginal bleeding. Per vaginal examination showed a whitish polypoid mass protruding through the cervix which appeared to originate from the uterine body. Magnetic resonance imaging (MRI) revealed an intravaginal polypoid tumor originating from the lateral wall of the uterus. The biopsy from the polypoid mass revealed a leiomyosarcoma. Therefore, the patient underwent a total abdominal hysterectomy with bilateral salpingo-oophorectomy. The histological examination revealed a biphasic tumor composed of benign and dilated endometrial glandes and malignant mesenchymal components which were composed of fascicles of spindle shaped cells with mild to moderate nuclear atypia and many mitosis. There are with areas of periglandular cuffs made by small round cells around glands. Immunohistochemical stains were performed and showed that round cells were positive for CD10, while spindle shaped cells were stained with AML and desmine. Histopathological final diagnosis was adenosarcoma with low-grade sarcomatous overgrowth and extensive smooth muscle differentiation. The patient has no evidence of disease, and she is doing well 18 months after the surgery.

Conclusion: We report a rare case of Uterine adenosarcoma with low gradeovergrowth and smooth muscle differentiation mimicking leiomyosarcoma.

Keywords: Adenosarcoma; Low Grade Overgrowth; Smooth Muscle Differentiation; Mixed Mullerian Tumors

\section{Introduction}

Uterine adenosarcoma is an uncommon malignant tumor, first described by Clement and Scully in 1974. It was defined as “...mixed tumors of the uterus, in which the stromal component has been malignant, but the epithelial elements, benign [1]. This tumor occurs most frequently in postmenopausal women [2]. However, 
exceptional cases have been reported in children [3]. The pathologic diagnosis is based on characteristic morphologic features with Immunohistochemical study. We here report a case of adenosarcoma mimicking a leiomyosarcoma of uterine corpus.

\section{Case Presentation}

A 59-year-old lady postmenopausal for more than 10 years, with vaginal bleeding. She had no past medical or surgical history. Per vaginal examination showed a large polypoid and whitish mass protruding through the cervix which seemed to originate from the uterine body. Magnetic resonance imaging (MRI) revealed an intravaginal polypoid tumor of $87 \times 70 \mathrm{~mm}$ originating from the lateral wall of the uterus (Figure1). A smooth Muscle Tumor (Leiomyoma or leiomyosarcoma) was suspected. A biopsy from the polypoidmass was performed with immunohistochemical study. Histopathological diagnosis was a leiomyosarcoma. Serum levels of CA19-9, CA125 and CEA were normal. The patient underwent a total abdominal hysterectomy with bilateral salpingo-oophorectomy. Gross examination of surgical specimen revealed a polypoid whitish mass measuring7x $8 \mathrm{~cm}$ protruding through the cervix and arising from the lateral wall of uterine corpus. It was firm with cystic areas. There was no macroscopic invasion of the myometrium.
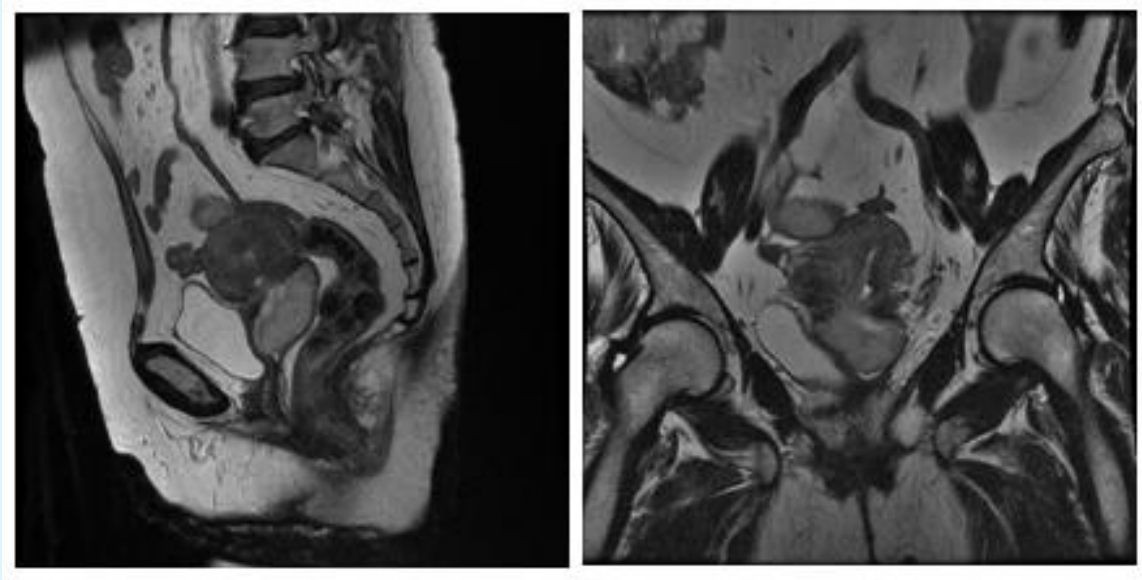

Figure 1: MRI imaging for adenosarcoma (A and B): both transverse plane and coronal plane showed anintravaginal polypoid tumor originating from the lateral wall of the uterus.

Histologically, the tumor was made by predominantly mesenchymal component (more than 25\%) which was composed of fascicles of spindle shaped (Figure 2) cells with mild to moderate nuclear atypia. Mitosis exceeded 40 per 10 high power fields. Extensive sampling showed benign dilated endometrial glandular elements with periglandular cuffs made by areas small round cells (Figure3\&4). No myometrial or lympho-vascular invasion was seen. Immunohistochemical stains were performed and showed that round cells were positive for CD10, while spindle shaped cells were stained with AML and desmine. Histopathological final diagnosis was adenosarcoma with extensive smooth muscle differentiation and low-grade sarcomatous Overgrowth. The patient has no evidence of disease 18 months after the surgery and she is doing well.

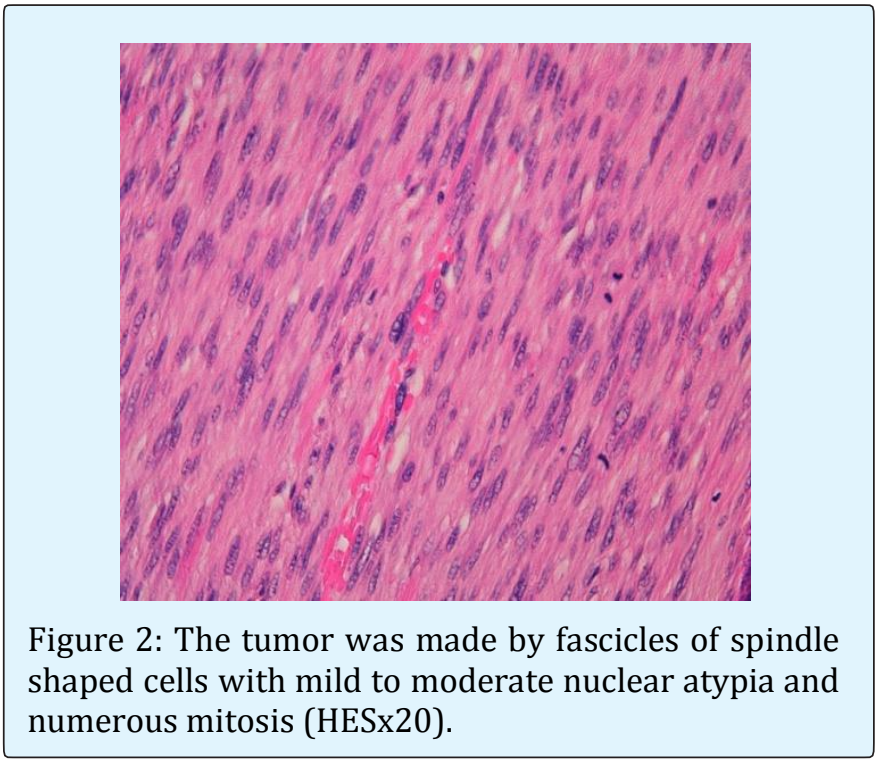




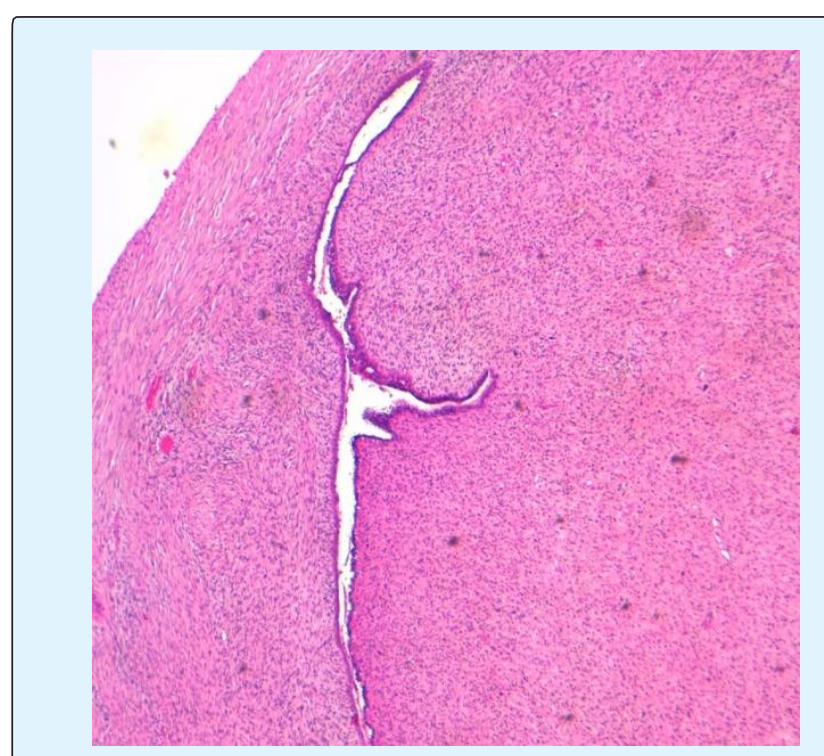

Figure 3: Benign dilated endometrial glandular element with predominate mesenchymal component (HEx5).

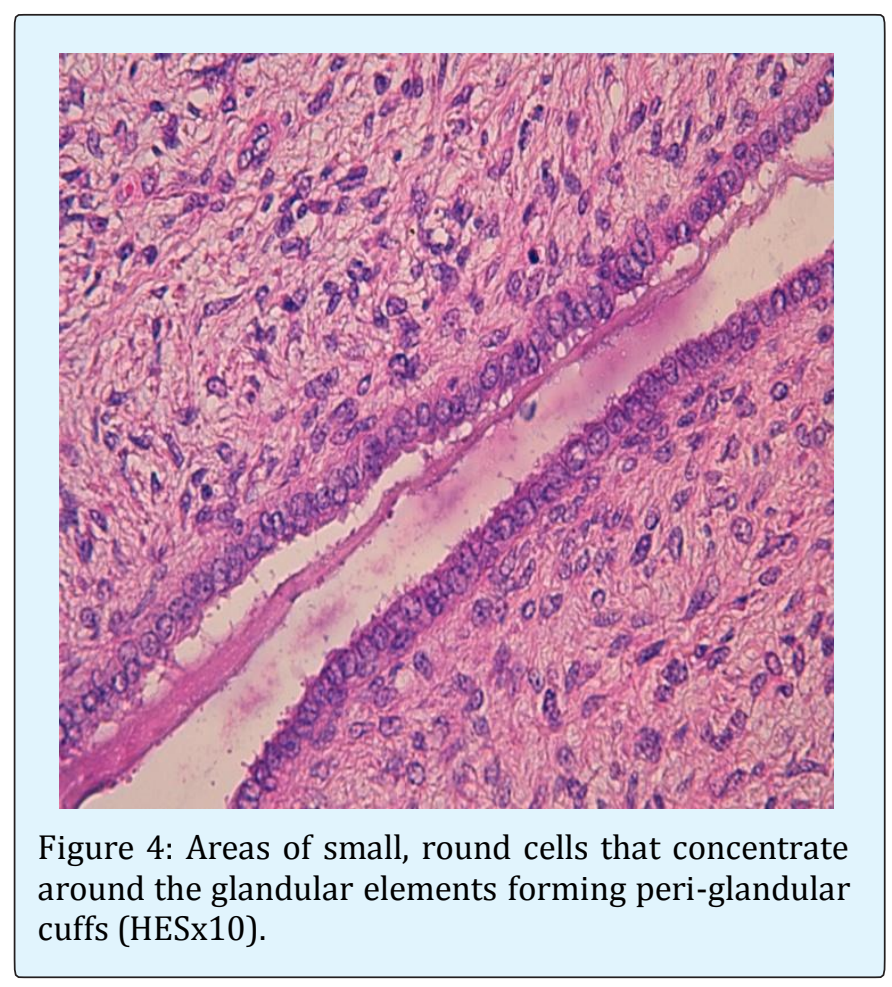

\section{Discussion}

Adenofibroma, adenosarcoma and carcinosarcoma are the three types of uterine mixed epithelial-mesenchymal tumors. Adenofibroma is composed of benign glandular elements and benign mesenchymal stroma, whereas carcinosarcoma is composed of malignant epithelial elements and mesenchymal stroma. Adenosarcoma is a rare entity which has benign glandular elements and malignant stroma [4], representing 3 to $9 \%$ of uterine cancers [5]. Some studies reported that estrogen stimulation; the use of tamoxifen, ororal contraceptive pill may increase the risk of Adenosarcoma [6-8].

In the current case, patient had no history of Longterm hormone therapy. The most common clinical symptoms are genital bleeding, pelvic pain or demonstration of a pelvic mass. MRI is the imaging modality of choice to examine the local extent of disease. It shows a large uterine mass that is hypointenseon $\mathrm{T} 1$, with cystic appearance on T2 [5]. It typically presents as an exophytic or polypoid mass, originating from the uterine wall or fundus which protrudes from the cervix [4].

Such tumor is usually observed in the endometrium, but can arises in the cervix, ovaries, and fallopian tubes.

Histologically, adenosarcoma is composed of benign glandular elements and malignant stroma that may compress the benign epithelium, and then resembling aphyllodes tumor of the breast. Condensation of small stromal cells around dilated glandes is characteristic and it is called Periglandular cuffing. The epithelium may show mild to moderate atypia and is usually endometrioid. Themesenchymal component is classically a low-grade spindle cell sarcoma with mitoses (at least 2 per 10 high-powerfields). The main histological prognostic factors are sarcomatous overgrowth, and deep myometrial invasion.

Sarcomatous overgrowth is defined as the presence of pure mesenchymal component without any epithelial elements comprising at least $25 \%$ of the tumor. It typically consists on a high grade sarcomatous areas with severe cytologic atypia, high mitotic activity, and necrosis; it is associated with a poor outcome. However, rare cases of low grade sarcomatous overgrowth, as in our case have been reported $[9,10]$. In the current case, the majority the sampled tissue showed a low grade mesenchymal neoplasm with smooth muscle differentiation resembling a leiomyosarcoma with only a minor component showing a conventional adenosarcoma. This reason it was initially misinterpreted as a leiomyosarcoma on biopsy. Furthermore, Smooth muscle differentiation has rarely been reported in the literature [9,11-13] and consists typically in compact fascicles of smooth muscle cells as it was observed in our case. At immunohistochemical study, mesenchymal cells of 
adenosarcomas is typically stained by ER, PR, CD10, and WT1. Other markers that can be positive in adenosarcomas like vimentin, smooth muscle actin, desmin, CD34 , calretinin , and AE1/3 cytokeratin [14]. The Ki67 index is usually $<5 \%$ but more than $20 \%$ in areas of sarcomatous overgrowth or glandular cuffing.

Lymph node metastasis rate is low, therefore staging lymphadenectomy is not recommended in patients with disease confined to the uterus and without high risk factors. No systemic or adjuvant treatment have been validated for adenosarcoma, howeverchemotherapy can be used in patent with adenosarcoma with sarcomatous overgrowth.

\section{Conclusion}

Mullerian Adenosarcoma should be considered in the differential diagnosis of polypoid mass protruding from the cervix. The final diagnosis is usually made on surgical specimen and it is based on characteristic morphologic features with benignepithelial component and malignant mesenchymal component.

Contributors: All authors contributed to the realization of this manuscript.

Competing Interest: No benefits in any form have been received or will be received from a commercial party related directly or indirectly to the subject of this article.

\section{References}

1. Pinto A, Howitt B (2016) Uterine adenosarcoma. Arch Pathol Lab Med 140(3): 286-290.

2. Singh R, Shameema S, Vijaya K, Kumar P (2010) Mullerian adenosarcoma of the uterus with sarcomatous overgrowth. Clinical Medicine Insights: Case Report 3: 27-30.

3. Fleming NA, Hopkins L, de Nanassy J, Senterman M, Black AY (2009) Mullerian adenosarcoma of the cervix in a 10-year-old girl: case report and review of the literature. Journal of pediatric and adolescent gynecology 22(4): e45-e51.

4. Taga S, Sawada M, Nagai A, Yamamoto D, Ryoji Hayase, et al. (2014) A Case of Adenosarcoma of the Uterus. Case reports in obstetrics and gynecology 342187.
5. Nathenson MJ, Ravi V, Fleming N, Wang WL, Conley A (2016) Uterine adenosarcoma: a review. Current oncology reports 18(11): 68.

6. Nomura K, Aizawa S, Ushigome S (2001) Adenosarcoma of the uterine corpus associated with ovarian thecoma. Pathology international 1(9): 735738.

7. Tjalma WA, Michener CM (2005) Mullerian adenosarcoma of the uterus associated with longterm oral contraceptive use. European Journal of Obstetrics \& Gynecology and Reproductive Biology 119(2): 253-254.

8. Wysowski DK, Honig SF, Beitz J (2002) Uterine sarcoma associated with tamoxifen use. New England Journal of Medicine 346(23): 1832-1833.

9. Wu RI, Schorge JO, Dal Cin P, Young RH, Oliva E (2014) Müllerian adenosarcoma of the uterus with low-grade sarcomatous overgrowth characterized by prominent hydropic change resulting in mimicry of a smooth muscle tumor. International Journal of Gynecological Pathology 33(6): 573-580.

10. Clement PB (1989) Mullerian adenosarcomas of the uterus with sarcomatous overgrowth. A clinicopathological analysis of 10 cases. The American journal of surgical pathology 13(1): 28-38.

11. Oliva E, Clement PB, Young RH, Scully RE (1998) Mixed endometrial stromal and smooth muscle tumors of the uterus: a clinicopathologic study of 15 cases. The American journal of surgical pathology 22(8): 997-1005.

12. Yilmaz A, Rush DS, Soslow RA (2002) Endometrial stromal sarcomas with unusual histologic features: a report of 24 primary and metastatic tumors emphasizing fibroblastic and smooth muscle differentiation. The American journal of surgical pathology 26(9): 1142-1150.

13. Fehmian C, Jones J, Kress Y, Abadi M (1997) Adenosarcoma of the uterus with extensive smooth muscle differentiation: ultrastructural study and review of the literature. Ultrastructural pathology 21(1): 73-79.

14. Soslow RA, Ali A, Oliva E (2008) Mullerian adenosarcomas: an immunophenotypic analysis of 35 cases. The American journal of surgical pathology 32(7): 1013-1021. 